INVESTIGACIÓN EDUCATIVA

\title{
Consideraciones sobre la formación basada en competencias y el enfoque Una Salud
}

\author{
Felipe $A E^{1 *}$, Chiapparrone $L^{1}$, Caselli $A^{2}$ \\ 1. Secretaría Académica, Facultad de Ciencias Veterinarias - Universidad Nacional del \\ Centro de la Provincia de Buenos Aires (FCV-UNCPB), Argentina.
}

2. Programa de Conservación Comunitaria del Territorio, FCV-UNCPB, Argentina.

* Correspondencia: Antonio Felipe, FCV-UNCPB, Los Talas 480, (7000) Tandil, Buenos Aires, Argentina. E-mail: aefelipe@vet.unicen.edu.ar

RESUMEN. El enfoque Una Salud requiere de una estrecha relación entre los profesionales veterinarios con otros actores y factores del medio donde desarrollan sus actividades. Este trabajo aborda los aspectos que deberían considerarse al incluir los dominios de competencias del enfoque Una salud en el contexto de la formación veterinaria. Se tratan rasgos de los diseños curriculares y analizan posturas en torno a la formación basada en competencias, para proponer la opción del modelo sistémico complejo. Para que los veterinarios logren incrementar su desempeño efectivo e impactar positivamente en sus territorios laborales, es necesario fortalecer su pensamiento sistémico sobre la interdependencia de la salud humana, animal y ambiental, así como su capacidad de moverse en diferentes entornos sociales, políticos, legales y culturales. Los profesionales de la medicina veterinaria deben ser competentes para diseñar, gestionar y evaluar las interacciones entre los seres humanos, los animales y su medio y conformar equipos multidisciplinarios. Las estrategias educativas para mejorar la comprensión de los estudiantes de los determinantes sociológicos y ecológicos de la salud, así como sus responsabilidades profesionales, no deberían ser un agregado a planes de estudio por demás disciplinares y fragmentados.

\begin{abstract}
SUMMARY. Considerations on competency-based training and the One Health approach. The One health approach requires a close relationship between veterinary professionals and other actors and factors in the environment where they carry out their activities. This paper addresses the aspects that should be considered when including the domains of the One Health approach competencies in the context of a veterinary study program. Features of the curricular designs are discussed and positions on competency-based training are analyzed in order to propose the option of the complex-systemic model. In order for veterinarians to be able to enhance their effective performance and positively impact on their working environments, it is necessary to strengthen their systemic thinking about the interdependence of human, animal and environmental health, as well as their ability to move in different social, political, legal and cultural environments. Veterinary medicine professionals must be competent to design, manage and evaluate interactions between humans, animals and their environment and to be part of multidisciplinary teams. Educational strategies to enhance students' understanding of the sociological and ecological determinants of health, as well as their professional responsibilities, should not be an add-on to too fragmented and disciplinary curricula.
\end{abstract}

Palabras clave: competencias, diseño curricular, estrategias didácticas, Una Salud

Keywords: competences, curriculum design, teaching strategies, One Health

\section{Introducción}

Los sistemas educativos en general y la educación universitaria en particular se han visto desafiados en las últimas décadas a innovar en sus estrategias de enseñanza y de aprendizaje desde diferentes sectores sociales. Las demandas de cambio se basan en los avances científicotecnológicos, nuevos requisitos del mundo laboral y modificaciones en el contexto de trabajo educativo como la actual pandemia de COVID-19
(Pérez Zúñiga et al., 2018; Dussel et al., 2020; Sanz et al., 2020). Un aspecto común de las demandas hacia la educación superior es centrar su accionar en la formación basada en competencias (Salas et al., 2016; Cejas Martínez et al., 2019; Giannini, 2020). El término competencias es polisémico y ha incrementado su ambigüedad en la misma medida en que se debaten sus interpretaciones e implicancias educativas (Zapata Callejas, 2015). La concepción de enseñanza basada en competencias no es ajena a esa diversidad conceptual ni a 
los distintos enfoques de la política educativa. En términos generales, podemos asumir que la enseñanza basada en competencias es un proceso complejo centrado en la construcción personal de los estudiantes para que, de manera gradual, adquieran, desarrollen y apliquen capacidades y habilidades para resolver situaciones tanto de su vida social como laboral (Marrero Sánchez y Lasso De La Vega González, 2017; Cejas Martínez et al., 2019). La educación basada en competencias presenta siete elementos centrales: 1- los estudiantes deben tomar decisiones sobre sus necesidades y experiencias de aprendizaje, la adquisición de conocimientos y su utilización; 2los estudiantes progresan mediante la demostración de dominio en distintos campos de acción; 3- se establecen para cada competencia objetivos de aprendizaje que deben poder medirse y ser transferibles, además de fortalecer las capacidades, las actitudes y el protagonismo de los estudiantes en uno o más grupos sociales; 4- la evaluación es formativa; 5- existe un acompañamiento continuo de los estudiantes durante todo el proceso para resolver sus necesidades de aprendizaje y 6 - los resultados de aprendizaje son capacidades, habilidades y disposiciones para aplicar los conocimiento adquiridos $y$, de ser necesario, reformularlos (Levine y Patrick, 2019; Evans et al., 2020).

La incorporación de la formación basada en competencias es una decisión de política educativa que se ha aplicado en numerosas innovaciones de diseños curriculares del nivel superior. La referencia a las competencias aparece dentro de una amplia diversidad de planes de estudio. Según el punto de partida desde el cual se enfoca un diseño curricular, discriminan entre: 1- quienes consideran los problemas profesionales sin que éstos se vinculen en su proceso de resolución con la complejidad del contexto y los objetos de trabajo, 2- los que extrapolan diseños curriculares exitosos ya implementados sin contemplar la realidad local, 3- los que asumen planes de estudio estáticos, sin dialéctica curricular, por lo tanto la innovación es cambiar de ubicación, carga horaria o denominación a una asignatura, 4- los centrados en competencias sin una discusión profunda de su origen e implicancias y 5- los que parten de la actividad profesional como modeladora de "todas las acciones del sujeto donde se manifiestan, no sólo las relaciones sociales de producción, también las relaciones que el profesional crea en función de su subsistencia" (Cabrera Marrero et al., 2017).
Este trabajo se orienta a abordar los aspectos que deberían considerarse al trabajar con el enfoque Una Salud y sus dominios de competencias, en el contexto de la formación veterinaria. Las consideraciones que se presentan son potencialmente transferibles para otros enfoques transversales.

\section{Diseños curriculares y formación basada en competencias}

El enfoque por competencias impulsó, por diferentes razones, a que el sistema universitario de las últimas décadas orientara parte de su trabajo a intentar adaptarse al aparato productivo y a los avances tecnológicos, más que a las necesidades sociales. Así, en un principio, se entendía a la formación por competencias como la preparación para el mundo laboral. Paulatinamente, se gestó la idea de la formación integral centrada en los estudiantes, proponiendo crear diseños curriculares que derivaran en planes de estudio integrados, flexibles y dinámicos, con capacidad para ajustarse a las diversas y cambiantes necesidades educativas y de generar recursos didácticos innovadores (Freire Quintana et al., 2018). Organizar, articular e integrar contenidos en los diseños curriculares, en especial de las carreras biomédicas y tecnológicas, además de un enorme desafío, genera tensiones que deben ser resueltas al interior de cada comunidad educativa (Verna, 2011).

La promoción de la enseñanza basada en competencias en las instituciones de educación superior argentinas reconoce dos factores: la necesidad de funcionalidad de los aprendizajes y la función social de la enseñanza, basada en: "...formar en todas las capacidades del ser humano con el fin de dar respuesta a los problemas que depara la vida, se convierte, así en la finalidad primordial de la escuela; formación integral de la persona como función básica en lugar de la función propedéutica, una escuela que forme en toda aquellas competencias imprescindibles para el desarrollo personal, interpersonal, social y profesional" (Trujillo-Segoviano, 2014, p. 311). Esta expresión coincide con las premisas del enfoque Una Salud, que se abordan en el apartado La concepción de Una Salud, ya que el mismo requiere de la formación integral de profesionales con capacidades y habilidades para comprender y transformar el entorno. Por consiguiente, el desarrollo de diseños curriculares donde se adopte tal enfoque debiera incorporar una formación basada en competencias básicas, 
técnicas, disciplinarias específicas y blandas o sociales (Atusingwize et al., 2020; Tungthongchai et al., 2018). Este requisito es común para otros enfoques transversales como los de bienestar animal, bioseguridad y desarrollo sostenible, por cuanto todos se vinculan para lograr la formación integral de los estudiantes de carreras con fuerte impacto social, como la medicina veterinaria.

Cuando se discuten los modelos de diseños curriculares más adecuados para la formación profesional, se recurre al análisis de los niveles de integración curricular en medicina humana basado en la escalera de Harden (Escanero Marcén, 2007) y, más recientemente, en el Modelo Educativo para Desarrollar Actividades Profesionales Confiables (MEDAPROC) (HamuiSutton et al., 2018 y 2019; Francischetti et al., 2020; Pinilla et al., 2021). A medida que se asume un diseño curricular más integrado, con una visión profesionalizante no tradicional, centrado en los estudiantes, la concepción del enfoque por competencias va modificándose. No está de más señalar, considerando a los detractores del modelo por competencias, que no hay posibilidad alguna de adquirir y enriquecer competencias sin contenidos, sin conocimientos disciplinares sólidos que le den sustento a las capacidades y habilidades. Pero tampoco es sencilla la adquisición de competencias en planes de estudio lineales y fragmentados, aunque por corrección política se las enuncie. Tal como el uso cotidiano del constructo "aprendizaje significativo", el de formación por competencias, se ha vuelto excesivamente frecuente como una referencia obligada desde la década de los '90 en nuestro país. Excede los límites de este artículo asumir tales discusiones. Nos centraremos en aspectos puntuales de la formación por competencias en tanto referidas a la adopción del enfoque Una Salud en los diseños curriculares de ciencias veterinarias.

\section{Competencias en la formación veterinaria}

Durante el siglo XX, las innovaciones curriculares hacia modelos de diseños por competencias surgieron en medicina humana (Mantilla et al., 2021). La educación veterinaria basada en competencias es un enfoque modelado a partir de las propuestas vigentes en la educación médica (AAVMC, 2018), actualizado periódicamente por el Grupo de Trabajo Sobre Educación Veterinaria Basada en Competencias (Asociación Americana de Colegios Médicos Veterinarios - AAVMC, 2018). Se dispone de una amplia serie de artículos y documentos referidos a competencias profesionales en medicina veterinaria como la edición de la Organización Panamericana de la Salud (OPS), la Organización Mundial de la Salud (OMS) y la AAVMC sobre la Visión del Futuro de la Educación Médica Veterinaria (AAVMC, 2007), otros publicados por la Asociación Panamericana de Ciencias Veterinarias (PANVET) y la Federación Panamericana de Facultades y Escuelas de Ciencias Veterinarias (Taylor Preciado, 2012), del libro Perfil Profesional del Médico Veterinario en Latinoamérica, Visión al 2030 (Taylor Preciado, 2013), y las recomendaciones y directrices de la Organización Mundial de Sanidad Animal (OIE) sobre las competencias de los veterinarios recién licenciados y paraprofesionales de veterinaria (OIE, 2012 y 2018), entre otros. En cuanto a los diseños curriculares centrados en la adquisición de competencias en ciencias veterinarias, existen análisis de las experiencias desarrolladas en el Espacio Europeo de Educación Superior (Rouco et al., 2009), la Argentina (Galván et al., 2011; Cánepa, 2016), México (Valle Flores, 2014), España (Climént Bonilla, 2014; DavóBlanes et al., 2018) y Colombia (Contreras Castro, 2015). En el caso particular de la Argentina, las carreras de Veterinaria y Medicina Veterinaria se rigen por la Resolución 1034/2005 del Ministerio de Cultura y Educación, donde aparece el término competencias en relación con los contenidos curriculares básicos. En el texto se señala que tales contenidos: "Comprenden no sólo la información conceptual y teórica considerada imprescindible, sino las competencias que se desean formar."

\section{La concepción de Una Salud}

Una Salud es un enfoque transversal que reconoce que la salud de las personas está estrechamente relacionada con la salud de los animales y el medio ambiente que comparten. Por este motivo se concibe a Una Salud como un enfoque colaborativo, multisectorial y transdisciplinario, que promueve el trabajo a nivel local, regional, nacional y global, con el objetivo de lograr resultados óptimos a partir del reconocimiento de la interconexión entre las personas, los animales, las plantas y su entorno compartido (Mackenzie y Jeggo, 2019; CDC's One Health Office, 2021; One Health Commission, 2021). Concebir Una Salud como un enfoque transversal conduce al desarrollo de acciones conjuntas entre diferentes sectores que abarcan la salud pública, la educación, la política, la legislación y la investigación para abordar las inequidades ambientales y sanitarias de manera integral y promover el acceso equitativo a los servicios y productos de salud, así como fomentar una gestión sostenible de los recursos naturales y los ecosistemas (Alianza Tripartita, 2019). En términos de Lerner y Berg (2015 y 2017), Una Salud es “... uno de los enfoques más amplios... incluye salud ambiental, ecología, medicina veterinaria, salud pública, medicina humana, molecular y microbiología, así como economía de la salud." Whitmee et al. (2015) señalan que Una Salud representa una perspectiva para mejorar la salud y el bienestar mediante la prevención de 
riesgos y la mitigación de los efectos de las crisis que se originan en la interfaz entre los seres humanos, los animales y sus diversos entornos. La orientación de las definiciones anteriores se enmarca en lo que One Health Global Network llama un enfoque de "toda la sociedad" (Keune et al., 2017). Desde esta perspectiva todas las ciencias de la salud y las disciplinas relacionadas con ellas, trabajan a través de sus fronteras de manera colaborativa para mejorar la salud y alcanzar un nivel óptimo. Últimamente, ha habido un énfasis creciente dentro de la comunidad científica de Una Salud por ampliar su concepción abarcando la salud humana y animal, la biodiversidad, la ecología, el cambio climático, los sistemas agrícolas e incorporar de manera activa a diversas ciencias sociales (Keune et al., 2017).

El enfoque de Una Salud implica la actividad profesional en vínculo estrecho con otros actores y factores del medio donde se desarrolla. El concepto de Una Salud reconoce las conexiones de la salud entre los seres humanos, los animales y sus entornos compartidos y promueve la cooperación interprofesional de veterinarios, biólogos, médicos, sociólogos y otras profesiones para abordar problemas complejos que afectan la estabilidad de las poblaciones de especies en entornos cambiantes (Rabinowitz et al., 2018). Una Salud también fomenta el desarrollo del potencial de los veterinarios en relación con otras profesiones para lograr aprendizajes conjuntos a través de un enfoque comparativo y evolutivo de los problemas ambientales (Natterson-Horowitz, 2012). La adopción del enfoque Una Salud en los planes de estudio requiere formar a los estudiantes en distintos dominios de competencias. Su logro implica gestionar múltiples interacciones entre docentes, estudiantes, profesionales, comunidades y sus entornos compartidos. La incorporación de las competencias en Una Salud conlleva repensar la educación veterinaria con el diseño y la implementación de planes de estudio innovadores. Esta meta parte de cuestionar los planes actuales por considerar que forman graduados bien equipados técnicamente pero con una comprensión y habilidades muy limitadas de los aspectos sociológicos y ecológicos que implican sus profesiones (Mor et al., 2018)

\section{Competencias y enfoque Una Salud}

Los principios y las competencias de los profesionales para abordar el enfoque multisectorial Una Salud y su inserción educativa han sido definidos para distintas regiones del planeta mediante debates internacionales (Fenwick, 2016; Alianza Tripartita FAO, OIE y OMS, 2019; Amuguni et al., 2019; Ferri y Lloyd-Evans, 2021; Gruetzmacher et al., 2021). La Alianza Tripartita FAO, OIE y OMS se refiere a la constitución de fuerzas laborales de Una Salud nacionales, integrales, multidisciplinarias y multisectoriales. Dichas fuerzas deben disponer de competencias técnicas y multisectoriales y poder desempeñarse en diversos ambientes institu- cionales para abordar problemas surgidos en la interfaz entre humanos, animales y ambiente (Alianza Tripartita, 2019).

Proponer la enseñanza basada en competencias para el enfoque Una Salud, requiere de clarificar la posición ideológica desde la cual se parte. Desde su origen, la educación por competencias reúne estrategias educativas que se centran en la adquisición y desarrollo de capacidades y habilidades orientadas al mundo laboral y, en general, asociado con la formación técnica y profesional. Becerra Gálvez y Campos Ahumada (2012) señalan que el enfoque por competencias principia hacia finales de la década de 1960, ante la necesidad de elaborar respuestas que optimizaran los vínculos entre los sistemas educativos y las necesidades y demandas del mundo laboral. Este origen marcó un debate que perdura con dos polos opuestos que pueden sintetizarse, de manera casi coloquial, en si la educación forma para el trabajo o para la vida. En la actualidad, persisten y se combinan distintos modelos para determinar cuáles son las competencias en las que debería centrarse la educación. Los más empleados en la identificación de competencias son el modelo conductual o conductista, el modelo funcional o funcionalista, el modelo constructivista (Delgado Bohórquez, 2009) y el modelo sistémico complejo (Tobón, 2007; Trujillo-Segoviano, 2014).

El modelo funcionalista se centra en identificar las competencias a través de las tareas desempeñadas en un determinado puesto de trabajo, observando y evaluando los resultados, pero no el proceso que permite obtenerlos. El modelo conductista se basa en observar, evaluar y medir el comportamiento de personas consideradas con desempeño óptimo para el logro de resultados en un puesto de trabajo y en un contexto concreto. Así las competencias se definen en términos de atributos personales (Díaz Vargas, 2020). El tercer modelo, constructivista, organiza las competencias considerando, además de las metas laborales, las potencialidades de cada sujeto y su entorno (Becerra Gálvez y Campos Ahumada, 2012). En este modelo, las competencias no sólo se identifican a partir de la función a desempeñar sino también de la relevancia de las personas (Díaz Vargas, 2020) y el entorno sociolaboral con el que se relacionan (Delgado Bohórquez, 2009). El modelo sistémico-complejo constituye una alternativa donde se prioriza la formación de personas desde una perspectiva integral, basado en el compromiso ético, contemplando su autoconocimiento, automotivación y la autoestima, las cuales puedan aportar a la sociedad siendo profesionales idóneos y emprendedores (Tobón, 2007; UNESCO, 2013). Este enfoque permite la gestión de un currículum por competencias y toma del constructivismo la relevancia de la participación de las personas no sólo en su adquisición de contenidos académicos sino también "... en la discusión y comprensión de los problemas, involucrando para ello a todos los protagonistas que conforman el tejido social de las 
organizaciones y de su entorno formativo" (Becerra Gálvez y Campos Ahumada, 2012, p. 17).

La opción del modelo sistémico complejo para la formación basada en competencias del enfoque Una Salud

En primer término, debe considerarse que el enfoque Una Salud abarca un amplio campo de disciplinas agrupables en las ciencias humanas, sociales, exactas, biológicas y médicas, de la ingeniería y tecnológicas (Lerner y Berg, 2015 y 2017). A su vez, implica la generación, el diseño y el desarrollo de encuadres integrales para prever, evitar y abordar las enfermedades de animales y humanos, considerando componentes ambientales, culturales, políticos y socioeconómicos (Bardosh et al., 2020; Michalon, 2020). Cabe considerar, entonces, que una mirada desde las prácticas educativas tradicionales no pareciera ser la primera opción frente a ese enfoque.

La Oficina Internacional de Educación (OIE-UNESCO, 2021) señala que esta organización define al enfoque de enseñanza por competencias como "la adquisición de conocimiento a través de la acción". El concepto de competencia se enfoca en los saberes de ejecución: saber pensar, saber interpretar, saber desempeñarse y saber actuar en diferentes escenarios. Al respecto afirma que el concepto de competencia es el pilar del desarrollo curricular y el incentivo tras el proceso de cambio. Se define como "el desarrollo de las capacidades complejas que permiten a los estudiantes pensar y actuar en diversos ámbitos [...]. Consiste en la adquisición de conocimiento a través de la acción, resultado de una cultura de base sólida que puede ponerse en práctica y utilizarse para explicar qué es lo que está sucediendo" (Braslavsky, C. citada en UNESCO, 2007). La elección de las competencias como principio organizador del curriculum es una forma de trasladar la vida real al aula (Jonnaert et al., 2006). Se trata, por tanto, de dejar atrás la idea de que el curriculum se lleva a cabo cuando los estudiantes reproducen el conocimiento teórico y memorizan hechos. Formar profesionales competentes y proactivos desde una perspectiva integral, supera a la educación transmisiva, máxime si consideramos que Una Salud implica para la Organización Mundial de Sanidad Animal la necesidad del trabajo colaborativo global. En consonancia con esta postura, la OMS se refiere a Una Salud como el enfoque multisectorial que permite diseñar y aplicar programas, políticas, leyes e investigaciones en el que múltiples sectores se comunican y colaboran para lograr mejores resultados de salud pública (OMS, 2017).

El modelo sistémico complejo pareciera ser el más adecuado para construir alternativas de diseños curriculares innovadores con enfoques integrales, como el de Una Salud. Este modelo implica rigurosidad en el estudio del contexto y la consideración de las competencias como elementos organizadores de los perfiles y mallas curriculares (Tobón, 2007). Sus rasgos específicos, según Tobón (2007) son: “1) las competencias se abordan desde el proyecto ético de vida de las personas, para afianzar la unidad e identidad de cada ser humano, y no su fragmentación; 2) las competencias buscan reforzar y contribuir a que las personas sean emprendedoras, primero como seres humanos y en la sociedad, y después en lo laboral empresarial para mejorar y transformar la realidad; 3) las competencias se abordan en los procesos formativos desde unos fines claros, socializados, compartidos y asumidos en la institución educativa, que brinden un para qué que oriente las actividades de aprendizaje, enseñanza y evaluación; 4) la formación de competencias se da desde el desarrollo y fortalecimiento de habilidades de pensamiento complejo como clave para formar personas éticas, emprendedoras y competentes; y 5) desde el enfoque complejo la educación no se reduce exclusivamente a formar competencias, sino que apunta a formar personas integrales, con sentido de la vida, expresión artística, espiritualidad, conciencia de sí, etc., y valores" (Tobón, 2007, p. 15).

El modelo sistémico complejo implica una forma de trabajo que conduce a considerar de manera multi e interdisciplinar los problemas surgidos o potenciados en las últimas décadas, en coincidencia con las premisas del enfoque Una Salud. Este enfoque nos desafía a proponer soluciones integrales frente a la globalización del comercio, del turismo, de la información y de las inversiones, el impacto de los sistemas alimentarios consolidados, de la urbanización y de impulsores antropogénicos que impactan negativamente en nuestros ecosistemas, creando una nueva interdependencia dinámica y sin precedentes entre la salud y el bienestar de las personas, los animales y el ambiente (Herrmann y Johnson-Walker, 2018; Rabinowitz et al., 2018). Esta interdependencia, que incluye factores sociales, económicos, políticos y biológicos, crea amenazas y riesgos para la salud, como la emergencia de enfermedades, la resistencia antimicrobiana y las enfermedades transmitidas por los alimentos, entre otros. Asimismo, produce cambios ecológicos que fracturan nuestro planeta, como la desertificación y el cambio climático (Herrmann y Johnson-Walker, 2018; Mackenzie y Jeggo, 2019).

\section{Discutiendo concepciones}

Al abordar las competencias en Una Salud, la Alianza Tripartita presenta una definición que remite a los modelos conductista y funcionalista. Se afirma que una competencia es: "Una característica compuesta de 3 elementos: técnica (habilidad para hacer algo), conocimiento (comprensión de un tema) y habilidad (talento adquirido para el desempeño) que, en conjunto, permiten a una persona ser eficaz y alcanzar un desempeño superior" (Alianza Tripartita, 2019, p. 120). La formación contemporánea de profesionales capaces de trabajar dentro del enfoque Una Salud, asumiendo el modelo sistémico complejo, va más allá del desempeño personal o profesional. 
La Alianza Tripartita FAO-OIE-OMS sostiene que el sector académico universitario dispone de los conocimientos y métodos como para desempeñar un papel decisivo en la formación de la fuerza laboral, tanto en lo que se refiere a las competencias técnicas específicas como a las propias de la aplicación del enfoque Una Salud. En el documento de la FAO-OIEOMS Adopción de un enfoque multisectorial "Una Salud" (Alianza Tripartita, 2019), en el ítem titulado Fuerza laboral "Una Salud" se adoptan indicaciones de la Organización Internacional del Trabajo en las cuales se destaca la necesidad de colaboración entre todos los sectores y disciplinas para lograr constituir una fuerza laboral eficaz. Señala además que: "Sin embargo, la mayoría de las estrategias nacionales y de los programas de educación y capacitación destinados al personal se centran en disciplinas y sectores específicos, con la consiguiente fragmentación y pérdida de eficacia del personal de salud cuando se la considera a nivel nacional. La adopción de un enfoque multisectorial "Una Salud" garantiza la creación de una fuerza de trabajo multidisciplinaria y multisectorial "Una Salud" para hacer frente a las enfermedades zoonóticas a nivel nacional” (Alianza Tripartita, 2019, p. 95).

La Alianza Tripartita pareciera orientar su propuesta específicamente a una fuerza laboral demandada por un determinado sistema. Al respecto, sugiere: "Elaborar programas de educación y capacitación para remediar las carencias del personal. Este paso se centra en el desarrollo de programas de educación y capacitación para remediar las carencias del personal de los sectores específicos y de "Una Salud"..." (Alianza Tripartita, 2019, p. 99). Una vez más, la complejidad inherente a Una Salud, hace difícil determinar cuál es ese sistema, ya que está en continuo desarrollo y, por consiguiente, demanda de profesionales con una formación que los dote de flexibilidad, comportamiento proactivo, autonomía, y otros atributos. Ahora bien, más allá de estas discrepancias, la Alianza Tripartita sugiere, para el diseño de programas de formación y capacitación, a las competencias básicas Una Salud elaboradas por la Comisión Una Salud (One Health Commission, 2021): gestión, comunicación e informática, valores y ética, liderazgo, trabajo en equipo y colaboración, funciones y responsabilidades y pensamiento sistémico. Cabría señalar las innovaciones de la Red Universitaria de Una Salud de África Central y del Este (OHCEA) que incorpora los dominios de cultura, creencias y género, política y promoción e investigación (Amuguni et al., 2019).

¿Qué implica la adopción del enfoque Una Salud y el trabajo basado en competencias en los planes de estudio? Sugerencias didácticas

Las premisas básicas que pueden considerarse para diseñar, desarrollar y evaluar actividades orientadas a la formación de competencias en Una Salud son comunes a las propuestas para las innovaciones educativas en e nivel superior en la última década (Roig Vila, 2018; Sánchez Mendiola et al., 2018; Rinaldi et al., 2021). En términos generales se orientan a la construcción de ambientes de aprendizaje y el diseño de planes de formación donde los métodos, recursos empleados, contenidos priorizados y contextos implicados en los procesos de enseñanza y de aprendizaje se centran en los estudiantes (Delgado Martínez, 2019). Tal como señala Meirieu (2007): “... no hace falta preguntarse antes de entrar en una clase qué diremos a los alumnos, hace falta preguntarse qué les haremos hacer para que aprendan alguna cosa, qué actividad les vamos a proponer para permitirles acceder a un saber y estar a su lado para ayudarlos y, a la vez, exigirles" (Meirieu, 2007, p. 46).

La adopción del enfoque Una Salud y la adquisición y el desarrollo de competencias asociadas implican el diseño y puesta en práctica de estrategias y actividades educativas específicas. La mayoría, si no todas ellas, contribuyen a la formación integral de los estudiantes. Entre las metodologías aplicables al desarrollo de competencias en Una Salud pueden citarse el aprendizaje conceptual, el aprendizaje basado en fenómenos, el aprendizaje basado en problemas, los ejercicios de sensibilización cultural, ciudadanía responsable y mentalidad global, actividades de pensamiento sistémico, las prácticas socioeducativas y la formación experiencial. A continuación se presentan algunos ejemplos de actividades.

El aprendizaje basado en problemas En este método los estudiantes deben utilizar estrategias de razonamiento para formular hipótesis explicativas y posibles alternativas de solución a una situación problema multi o interdisciplinar. Un aspecto relevante de este método. es que los estudiantes son motivados a identificar sus necesidades de aprendizaje relacionadas con conocimientos, habilidades y actitudes que se necesitan para abordar el problema, deben utilizar sus conocimientos previos y buscar nueva información para aplicarla, consultar a expertos, elaborar un plan de acción y coevaluar sus resultados. Los docentes construyen la situación problema con un contexto social y cultural que puede tener datos e información básica y establecen los objetivos generales de la actividad (Vega Ramírez et al., 2010). Durante todo el proceso los estudiantes trabajan en grupos colaborativos, son tutorados por sus docentes y emplean diferentes estrategias de razonamiento. EI modo de evaluación también se modifica. Además de implementar la evaluación formativa, los estudiantes deben ser desafiados a elaborar ensayos, presentaciones orales o portafolios (Putra et al., 2016).

Educación basada en la comunidad. Los estudiantes deben comprender los determinantes ambientales y sociológicos de la salud, así como sus futuras responsabilidades profesionales en áreas relacionadas directa 
o indirectamente con las ciencias veterinarias. Las prácticas de campo les permiten actuar como observadores de la interacción de las comunidades humanas con el entorno. Los espacios físicos no sólo pueden ser establecimientos productivos, sino reservas naturales, parques, localidades de reducido tamaño, etc. Parte del trabajo a realizar en estas experiencias es identificar la presencia de animales exóticos (por ejemplo, ganado doméstico) y asociarla con posibles enfermedades, el impacto de las culturas y los sistemas de creencias en las prácticas de manejo de la zona, cambios en el uso de la tierra y la biodiversidad en diferentes sectores, y la existencia o no de planes de manejo de zoonosis. Se espera que los estudiantes evalúen los roles y responsabilidades de los veterinarios en la sociedad, comparen los beneficios y riesgos asociados con las interacciones humano-animal $y$ animal-animal, y evalúen las contribuciones de diversos sectores y profesionales a la salud y el bienestar de los animales, el ser humano y el medio ambiente (Mor et al., 2018). El trabajo en y con comunidades puede orientarse a que los estudiantes enfrenten desafíos y deban hacer contribuciones para resolverlos, implementando intervenciones en el territorio relacionadas con la salud pública. En el proceso de interacción con diferentes personas ponen en práctica habilidades de comunicación, trabajo en equipo, colaboración y la ética del trabajo (Atuyambe et al., 2016; Buregyeya et al., 2021). La educación basada en la comunidad se corresponde con el desarrollo de programas de formación experiencial centrados en la aplicación de las competencias profesionales para interactuar con las comunidades locales (Berrian et al., 2020).

Cursos multidisciplinarios y aprendizaje interprofesional. La organización de cursos con diversas modalidades, en los cuales participen estudiantes de diferentes disciplinas en torno a la temática de Una Salud favorece pensar de manera innovadora sobre las soluciones a problemas sanitarios. Existen experiencias mediante las cuales se trabaja con estudiantes de diferentes carreras como odontología, medicina, nutrición, veterinaria y artes (Linder et al., 2020; Buregyeya et al., 2021) o con estudiantes de veterinaria y medicina (Wilkes et al., 2018) para promover aprendizajes conjuntos en torno a los factores de riesgo asociados con las enfermedades zoonóticas, contaminación del agua, desechos tóxicos, impacto del cambio climático y seguridad alimentaria (Wilkes et al., 2018). Estas acciones de educación interprofesional fortalecen la formación general, desarrollan habilidades de comunicación y mejoran la capacidad para contribuir a la conformación de equipos colaborativos de Una Salud (Winer et al., 2014).

Experiencias de aprendizaje colaborativo internacional. Además de las propuestas educativas centradas en comunidades locales o en grupos interdisciplinarios, existen acciones educativas internacionales como la "Iniciativa conjunta para la enseñanza y el aprendizaje sobre los desafíos de la salud mundial y una sola salud" (Vicente et al., 2021). Esta iniciativa implica el trabajo conjunto de estudiantes pertenecientes a seis instituciones de cuatro países diferentes, para construir un ambiente colaborativo de enseñanza y aprendizaje en línea. La propuesta responde a los dominios de competencias relacionados con las prácticas interculturales e interprofesionales.

\section{Conclusiones}

La adquisición y el desarrollo de competencia propias del enfoque Una Salud mediante el modelo sistémico complejo puede favorecer la asimilación significativa, por parte de los estudiantes, de las interconexiones de las personas, los animales y el medio ambiente $y$ fomentar un pensamiento multidisciplinario. Las recomendaciones de políticas educativas referidas a la necesidad de cambios curriculares que respondan a los nuevos desafíos ambientales y sanitarios mundiales exigen de la colaboración intersectorial, tanto para comprender como para gestionar soluciones alternativas. La educación superior en medicina veterinaria debería analizar si sus modelos de estructuras académicas y los planes de estudio actuales facilitan la educación interdisciplinaria, promueven las habilidades de colaboración, trabajo en equipo y comunicación, que son críticas para el enfoque Una Salud. Asumir esta y otras perspectivas que demandan de una formación integral, requiere de innovaciones que mejoren las sinergias entre diversos sectores.

Para tener un desempeño efectivo e impactar positivamente en el territorio donde los veterinarios trabajan, es necesario fortalecer su pensamiento sistémico sobre la interdependencia de la salud humana, animal y ambiental, así como su capacidad de moverse en diferentes entornos sociales, políticos, legales y culturales. Los profesionales de la medicina veterinaria deben ser competentes para diseñar, gestionar y evaluar las interacciones entre los seres humanos, los animales y su medio y conformar equipos multidisciplinarios proactivos. Al respecto, cabe destacar que Una Salud es un enfoque integrado, que trasciende las disciplinas, no una potencial asignatura más en la formación de grado. Las estrategias educativas para mejorar la comprensión de los estudiantes de los determinantes sociológicos y ecológicos de la salud, así como sus responsabilidades profesionales, no deberían ser un agregado a los planes de estudio por demás disciplinares y fragmentados. Antes bien, al ser Una Salud, por definición, un "enfoque transversal", debiera ser una perspectiva de trabajo en todas las asignaturas posibles, articulando actividades existentes o adaptando las mismas como prácticas interdisciplinares.

\section{Referencias}

AAVMC. 2007. Visión del Futuro de la Educación Médica Veterinaria. Journal of Veterinary Medical Education. 
Association of American Veterinary Medical Colleges Special Edition 2007 - 34. Disponible en: https://fmvz.unam.mx/fmvz/principal/archivos/Vision Futuro \%20Educacion Veterinaria.pdf

AAVMC. 2018. Working Group on Competency-Based Veterinary Education. Molgaard, L.K., Hodgson, J.L., Bok, H.G.J., Chaney, K.P., Ilkiw, J.E., Matthew, S.M., May, S.A., Read, E.K., Rush, B.R., Salisbury, S.K. (eds.). Competency-Based Veterinary Education: Part 1 - CBVE Framework. Washington, DC: Association of American Veterinary Medical Colleges.

Alianza Tripartita. 2019. Taking a Multisectoral, One Health Approach: A Tripartite Guide to Addressing Zoonotic Diseases in Countries. Published by The Food and Agriculture Organization of the United Nations, The World Organisation for Animal Health, The World Health Organization. Disponible en:

https://apps.who.int/iris/bitstream/handle/10665/325620/97 89241514934-eng.pdf?sequence $=1$ \&isAllowed $=y$

Amuguni H, Bikaako W, Naigaga I, Bazeyo W. 2019. Building a framework for the design and implementation of one health curricula in east and Central Africa: OHCEAs one health training modules development process. One Health 7: 100073.

Atusingwize E, Ndejo R, Tumukunde G, Buregyeya E, Nsamba $P$, Tuhebwe $D$, Kato $C D$, Naigaga I, Musoke D, Kabasa JD, Bazeyo W. 2020. Application of one health approach in training at Makerere University: experiences from the one health workforce project in Uganda. One Health Outlook 2: 23.

Atuyambe LM, Baingana RK, Kibira SPS, Katahoire A, Okello E, Mafigiri DK, Ayebare F, Oboke H, Acio C, Muggaga K, Mbalinda S, Nabaggala R, Ruzaaza G, Arubaku W, Mary S, Akera P, Tumwine JK, Peters DH, Sewankambo NK. 2016. Undergraduate students' contributions to health service delivery through community-based education: A qualitative study by the MESAU Consortium in Uganda. BMC Med. Educ 16: 123 .

Bardosh KL, de Vries DH, Abramowitz S, Thorlie A, Cremers L, Kinsman J, Stellmach D. 2020. Integrating the social sciences in epidemic preparedness and response: A strategic framework to strengthen capacities and improve Global Health security. Global Health 16: 120

Becerra Gálvez M, Campos Ahumada F. 2012. El enfoque por competencias y sus aportes en la gestión de recursos humanos. Memoria Facultad de Ciencias Sociales, Santiago, Chile. Disponible en:

http://repositorio.uchile.cl/bitstream/handle/2250/116913/M emoria\%20MBecerra\%20FCampos.pdf?sequence=1

Berrian AM, Wilkes M, Gilardi K, Smith W, Conrad P, Zielinska Crook P, Cullor J, Nyatanyi T, Smith M, Kazwala R, Mazet J. 2020. Developing a Global One Health Workforce: The "Rx One Health Summer Institute" Approach. EcoHealth 17: 222-232.

Buregyeya $E$, Atusingwize E, Nsamba $P$, Nalwadda C, Osuret J, Kalibala $P$, Nuwamanya R, Okech S, Ssekamatte T, Nitumusiima S, Wakabi T, Bikaako W, Yawe A, Naigaga I, Kagarama J, Kabasa JD, Bazeyo W. 2021. Lessons from a community based interdisciplinary learning exposure: benefits for both students and communities in Uganda. BMC Med. Educ. 21:5.

Cabrera Marrero I, Crespo Zafra L, Portuondo PadrónR. 2017. El diseño curricular desde la perspectiva de la actividad profesional. Transformación 13: 406-415.
Cánepa PA. 2016. Implementación del sistema O.S.C.E. para la evaluación de competencias clínicas en la carrera de Medicina Veterinaria. Trayectorias Universitarias 2: 43-49.

CDC's One Health Office. 2021. One Health Basics. Centres for Disease Control and Prevention. U.S. Department of Health \& Human Services. Disponible en: https://www.cdc.gov/onehealth/basics/index.html

Climént Bonilla JB. 2014. Un acercamiento a las competencias de la educación veterinaria en México y España. Rev. Mex. Agroneg. 34: 761-772.

Contreras Castro JH. 2015. La perspectiva de la complejidad en el modelo didáctico del programa de Medicina Veterinaria y Zootecnia de una institución universitaria en Colombia. Magister en Educación. Universidad Autónoma de Bucaramanga, Colombia. Disponible en: https://repository.unab.edu.co/bitstream/handle/20.500.1274 9/2296/2015 Tesis Jorge Humberto Contreras.pdf?sequence =1\&isAllowed $=\mathrm{y}$

Davó-Blanes MC, Vives-Cases C, Huerta B. 2018. Competencias y contenidos de salud pública en los programas del Grado en Veterinaria en las universidades españolas. Gac. Sanit. 32: 526532.

Delgado Bohórquez VM. 2009. El modelo de competencias laborales: base para la gestión del talento humano en las organizaciones. Económicas CUC 30: 25-38.

Delgado Martínez LM. 2019. Aprendizaje centrado en el estudiante, hacia un nuevo arquetipo docente. Enseñanza \& Teaching 37: 139-154.

Díaz Vargas E. 2020. Enfoque teórico de modelos de competencias para la formación de directivos. Ámbito Investigativo 5: 24-30.

Dussel I, Ferrante P, Pulfer D. (compiladores). 2020. Pensar la educación en tiempos de pandemia. Entre la emergencia, el compromiso y la espera. 1a ed. Ciudad Autónoma de Buenos Aires, UNIPE: Editorial Universitaria. 369 pp.

Escanero Marcén JF. 2007. Integración curricular. Educ. Méd. 10: 23-30.

Evans C, Worthen M, Marion S. 2020. How systems of assessments aligned with competency-based education can support equity. Vienna, VA: Aurora Institute. Disponible en: https://aurora-institute.org/wp-content/uploads/howsystems-of-assessment-aligned-with-competencybasededucation-can-support-equity-jan-2020-web.pdf

Fenwick S. 2016. CORE Competencies and One health - from theory to action. Usaid-One Health Workforce. Hanoi, April 25th. Disponible en:

http://nckh.huph.edu.vn/sites/nckh.huph.edu.vn/files/BAI\%20 6\%20Core\%20Competencies\%20and\%200H\%20-

\%20theory\%20to\%20action\%20Stan_0.pdf

Ferri M, Lloyd-Evans M. 2021. The contribution of veterinary public health to the management of the COVID-19 pandemic from a One Health perspective. One Health 12: 100230.

Francischetti I, Holzhausen Y, Peters H. 2020. The time has come for Brazil: translating Competence Based Medical 
Education into practice by Entrustable Professional Activities (EPAs). Interface (Botucatu). 24: e190455

Freire Quintana JL, Páez MC, Núñez Espinoza M, Narváez Ríos M, Infante Paredes R. 2018. El diseño curricular, una herramienta para el logro educativo. Rev Com SEECI 45: 75-86.

Galván S, Gastaldi R, Manuale M. 2011. Enseñanza y evaluación de competencias en medicina veterinaria. Aula Universitaria 1: 79-90.

Giannini S. 2020. COVID-19 y educación superior: De los efectos inmediatos al día después. Análisis de impactos, respuestas políticas y recomendaciones. Internacional de la UNESCO para la Educación Superior en América Latina y el Caribe (UNESCO IESALC). 57 pp.

Gruetzmacher K, Karesh WB, Amuasi JH, Arshad A, Farlow A, Gabrysch S, Jetzkowitz J, Lieberman S, Palmer C, Winkler AS, Walzer C. 2021. The Berlin principles on one health - Bridging global health and conservation. Sci. Tot. Environ. 764: 142919.

Hamui-Sutton A, Durán-Pérez VD, García-Téllez S, Vives-Varela T, Millán-Hernández M, Gutiérrez-Barreto SE. 2018. Avances del Modelo Educativo para Desarrollar Actividades Profesionales Confiables (MEDAPROC). Educ. Méd. 19: 294 300.

Hamui-Sutton A, Vives-Varela T, Durán-Pérez V, GutiérrezBarreto S, Millán-Hernández M. 2019. Mapping an EPA-based comprehensive curricular proposal for UME. MedEdPublish 8: 37.

Herrmann JA, Johnson-Walker YJ (Eds.). 2018. Beyond One Health: from recognition to results. 1ra ed. John Wiley \& Sons, New Jersey: EEUU. 368 pp

Jonnaert P, Barrette J, Masciotra D, Yaya M. 2006. Revisión de la competencia como organizadora de los programas de formación: hacia un desempeño competente. Ginebra: Oficina internacional de Educación, BIE /UNESCO. 38 pp.

Keune $\mathrm{H}$, Flandroy L, Thys S, De Regge N, Mori M, AntoineMoussiaux N, Vanhove MPM, Rebolledo J, Van Gucht S, Deblauwe I, Hiemstra W, Häsler B, Binot A, Savic S, Ruegg SR, De Vries S, Garnier J, van den Berg T. 2017. The need for European OneHealth/EcoHealth networks. Arch. Public Health 26: 64 .

Lerner H, Berg C. 2015. The concept of health in One Health and some practical implications for research and education: what is One Health? Infect. Ecol. Epidemiol. 5: 25300.10.3402/iee.v5.25300

Lerner H, Berg C. 2017. A Comparison of Three Holistic Approaches to Health: One Health, EcoHealth, and Planetary Health. Front. Vet. Sci. 4: 163.

Levine E, Patrick S. 2019. What is competency-based education? An updated definition. Vienna, VA: Aurora Institute.

Linder D, Cardamone C, Cash SB, Castellot J, Kochevar D Dhadwal S, Patterson E. 2020. Development, implementation, and evaluation of a novel multidisciplinary one health course for university undergraduates. One Health 9: 100121.
Mackenzie JS, Jeggo M. 2019. The One Health approach. Why is it so important?. Trop. Med. Infect. Dis. 4: 88

Mantilla GC, Ariza K, Santamaría A, Moreno S. 2021. Educación médica basada en competencias: revisión de enfoque. Universitas Medica 62: 1-12.

Marrero Sánchez O, Lasso De La Vega González MC. 2017. El proceso de enseñanza-aprendizaje por competencias. Una visión desde el enfoque sistémico. Congreso Universidad 6: 2846.

Meirieu P. 2007. Es responsabilidad del educador provocar el deseo de aprender. Cuad. Pedagog. 373: 42-47.

Michalon J. 2020. Accounting for One Health: Insights from the social sciences. Rendre compte de One Health: réflexions issues des sciences sociales. Parasite 27: 56

Mor SM, Norris JM, Bosward KL, Toribio JLML, Ward MP, Gongora J, Vost M, Higgins PC, McGreevy PD, White PJ, Zaki S. 2018. One health in our backyard: Design and evaluation of an experiential learning experience for veterinary medical students. One Health 5: 57-64.

Natterson-Horowitz B. 2012. Zoobiquity: the astonishing connection between human and animal health. Ed. A.A. Knopf, New York, EEUU. 308 pp

OIE. 2012. Recomendaciones de la OIE sobre las competencias mínimas que se espera de los veterinarios recién licenciados para garantizar Servicios Veterinarios Nacionales de calidad. Disponible en: http://www.oie.int/fileadmin/Home/esp/

OIE. 2018. Directrices de la OIE sobre las competencias de los paraprofesionales de veterinaria. Disponible en: https://www.oie.int/fileadmin/Home/esp/Support to OIE M embers/Edu_Vet_AHG/E-Competence.pdf

OIE-UNESCO. 2021. Oficina Internacional de Educación. Enfoque por competencias. Disponible en: http://www.ibe.unesco.org/es/temas/enfoque-porcompetencias

OMS. 2017. El enfoque multisectorial de la OMS «Una salud». Organización Mundial de la Salud: Suiza. Disponible en: https://www.who.int/features/qa/onehealth/es/

One Health Commission. 2021. One Health Education - US Initiative. Disponible en: https://www.onehealthcommission.org/en/programs/one he alth education us initiative/

Pérez Zúñiga R, Mercado Lozano P, Martínez García M, Mena Hernández E, Partida Ibarra JÁ. 2018. La sociedad del conocimiento y la sociedad de la información como la piedra angular en la innovación tecnológica educativa. RIDE 8 16: 847870 .

Pinilla ES, Lenouvel A, Cantisani S, Klöppel W, Strik S, Huwendiek M, Nissen C. 2021. Working with entrustable professional activities in clinical education in undergraduate medical education: a scoping review. BMC Med. Educ. 21: 172.

Putra TA, Hezmee MN, Farhana NB, Hassim HA, Intan-Shameha AR, Lokman IH, Hamali AY, Salisi MS, Ghani AA, Shahudin MS, Qayyum MA, Hafandi A, Speare R, Fenwick SG. 2016. The application of One Health concept to an outdoor problem- 
based learning activity for veterinary students. Vet. World 9: 955-959.

Rabinowitz PM, Pappaioanou M, Bardosh KL, Conti L. 2018. A planetary vision for one health. BMJ Global Health 3: e001137.

Rinaldi SM, Durand MC, Salas Urdaneta EV. 2021. Apuntes sobre la innovación educativa en el nivel universitario. Universidad del CEMA. UCEMA, Buenos Aires, Argentina. Disponible en:

https://ucema.edu.ar/publicaciones/download/documentos/7 $\underline{94 . p d f}$

Roig Vila R. (coord.). 2018. El compromiso académico y social a través de la investigación e innovación educativas en la Enseñanza Superior. Editores Octaedro, Barcelona, España. $1256 \mathrm{pp}$.

Rouco A, Carrasco L, Goyache J. 2009. Estudios de Veterinaria en el Espacio Europeo de Educación Superior: El Proceso de Bolonia. Prof. Vet. 72: 6-10.

Sánchez Mendiola $M$, de los Santos J, Sánchez Saldaña MS 2018. ¿Qué es la innovación en educación superior? Reflexiones académicas sobre la innovación educativa. En Sánchez Mendiola M, Escamilla de los Santos J. (Coords.) Perspectivas de la innovación educativa en universidades de México: Experiencias y reflexiones de la RIE 360. Ciudad de México, México. Pp. 19-42. Disponible en: https://cuaieed.unam.mx/descargas/investigacion/Perspectiva s-de-la-innovacion-educativa-en-universidades-de-Mexico.pdf

Sanz I, Sáinz J, Capilla A. 2020. Efectos de la crisis del coronavirus en la educación. Organización de Estados Iberoamericanos para la Educación, la Ciencia y la Cultura (OEI), Área de Educación Superior, Ciencia y ETP, Madrid, España. 22 pp.

Taylor Preciado J. (Ed.). 2012. Competencias profesionales en Medicina Veterinaria. PANVET, Santiago, Chile. 152 pp.

Taylor Preciado J. (Ed.). 2013. Perfil profesional del Médico Veterinario en Latinoamérica. Visión 2030. PANVET, Amateditorial, Guadalajara, México. $81 \mathrm{pp}$

Tobón S. 2007. El enfoque complejo de las competencias y el diseño curricular por ciclos propedéuticos. Acción Pedagógica 16: $14-28$

Trujillo-Segoviano J. 2014. El enfoque en competencias y la mejora de la educación. Ra Ximhai 10: 307-322.

Tungthongchai $\mathrm{O}$, Prabpai $\mathrm{S}$, Amavisit $\mathrm{P}$, Phimpraphai W, Keowmookdar N, Pitiporntapin S, Butkatunyoo O, Hatthasak M, Ketkosan N. 2018. Integrating one health core competencies for multi-disciplines workforce program: a report. Kasetsart Educ. Rev. 22: 187-193.

UNESCO. 2007. Enfoque por Competencias, Oficina Internacional de Educación. Disponible en: http://www.ibe.unesco.org/es/comunidades/comunidad-depracticacop/enfoque-por-competencias.html

UNESCO. 2013. Las competencias en el desarrollo curricular. Disponible en:

http://www.ibe.unesco.org/cops/Competencies/Competencia s esp.pdf.Acceso en 01
Valle Flores A. (Coord.). 2014. Formación en competencias y certificación profesional. Universidad Nacional Autónoma de México. Metepec, México. Disponible en: http://132.248.192.241/ editorial/wpcontent/uploads/2018/06/Formaci\%C3\%B3n\%20en\%20compe tencias\%20y\%20certificaci\%C3\%B3n\%20profesional.pdf

Vega Ramírez FA, López Liria R, Padilla Góngora D, Santiuste Bermejo E, Martínez Cortés M, Rodríguez Martín C. 2010. El Aprendizaje Basado en Problemas en Ciencias de la Salud. R. Psicol. Educ. 1: 187-200.

Verna M. 2011. La hibridación como posibilidad para lograr un alto grado de flexibilidad curricular. Argonautas 1: 99-115.

Vicente $C R$, Jacobs $F$, de Carvalho DS, Chhaganlal $K$, de Carvalho RB, Raboni SM, Qosaj FA, Tanaka LF. 2021. Creating a platform to enable collaborative learning in One Health: The Joint Initiative for Teaching and Learning on Global Health Challenges and One Health experience. One Health 12: 100245.

Whitmee S, Haines A, Beyrer C, Boltz F, Capon AG, de Souza Dias BF, Ezeh A, Frumkin $H$, Gong $P$, Head $P$, Horton R, Mace GM, Marten R, Myers SS, Nishtar S, Osofsky SA, Pattanayak SK, Pongsiri MJ, Romanelli C, Soucat A, Vega J, Yach D. 2015. Safeguarding human health in the Anthropocene epoch: report of The Rockefeller Foundation-Lancet Commission on planetary health. Lancet 386: 1973-2028.

Wilkes MS, Conrad PA, Winer, JN. 2018. One Health-One Education: Medical and Veterinary Inter-Professional Training. J. Vet. Med. Educ. 46: 1-7.

Winer JN, Nakagawa K, Conrad PA, Brown L, Wilkes M. 2015. Evaluation of medical and veterinary students' attitudes toward a one health interprofessional curricular exercise. J. Interprof. Care 29: 49-54.

Zapata Callejas J. 2015. El modelo y enfoque de formación por competencias en la Educación Superior: apuntes sobre sus fortalezas y debilidades. Rev. Acad. Virt. 8: 24-33. 